\title{
Theories and applications of the inverse fractional natural transform method
}

\author{
Mahmoud S. Rawashdeh ${ }^{1 *}$ and Hadeel Al-Jammal ${ }^{1}$
}

${ }^{*}$ Correspondence:

msalrawashdeh@just.edu.jo

${ }^{1}$ Department of Mathematics and

Statistics, Jordan University of

Science and Technology, Irbid, Jordan

\begin{abstract}
In this paper, we propose a new method called the inverse fractional natural transform method (IFNTM). We present theoretical results and apply them to obtain approximate solutions of linear fractional ordinary differential equations (LFODEs) and partial differential equations (LFPDEs). The fractional derivatives are described in the Caputo sense. The algorithm described in this study is expected to be further employed to solve similar linear problems in fractional calculus.
\end{abstract}

MSC: 35J10; 33E12; 35A22; 44A10; 44A30; 81V10; 35C05; 35C10

Keywords: Fractional derivative; Caputo derivative; Natural transform

\section{Introduction}

In recent years, interest in the fractional differential equations has been stimulated due to their wide applications [1-4] in various fields of engineering and science $[7,8,12,17$, 20]. Various vital phenomena in electromagnetics, viscoelasticity, fluid mechanics, electrochemistry, biological population models, and signal processing are well described by fractional differential equations $[5,6]$. Also, they are employed in social sciences such as food supplement, climate, finance, and economics $[9,11,13,21,25,28]$. As a result, the importance of obtaining exact or approximate solutions of fractional linear and nonlinear differential equations in physics and applied mathematics is still a significant problem that needs new methods.

Also, a considerable interest has been shown in the so-called fractional calculus [20, 22], which allows us to consider integration and differentiation of any order, not necessarily integer. To a large extent, this is due to applications of the fractional calculus to problems in different areas of physics and engineering [26]. Moreover, in many applications, fractional calculus provides more accurate models of physical systems than ordinary calculus does.

The backward Kolmogorov equations in fractal space-time are based on the construction of a model for dynamic trajectories. The telegraph equation is a linear partial differential equation that describes the voltage and current on an electrical transmission line with distance and time.

Recently, many robust and efficient methods have been proposed to obtain approximate analytical solutions of fractional differential equations such as the fractional complex transform method [19], fractional Adomian decomposition method (FADM) [10, 30], fractional natural decomposition method (FNDM) [26, 29], the FRDTM [23, 24, 27], fractional

(c) The Author(s) 2018. This article is distributed under the terms of the Creative Commons Attribution 4.0 International License (http://creativecommons.org/licenses/by/4.0/), which permits unrestricted use, distribution, and reproduction in any medium, provided you give appropriate credit to the original author(s) and the source, provide a link to the Creative Commons license, and indicate if changes were made. 
Laplace decomposition method (FLDM) [15], and fractional Sumudu transform method (FSTM) [14, 18]. Finally, the natural transform method (NTM) was introduced by Khan et al. [16], who gave some properties and applications of the natural transform, which they called the N-transform.

In this paper, we give approximate analytical and exact solutions to two linear fractional differential equations: First, consider the linear fractional initial value problem of the form [19]

$$
D^{1 / 2} f(t)+f(t)=0,
$$

subject to the initial condition

$$
\left[D^{-1 / 2} f(t)\right]_{t=0}=2 .
$$

Second, consider the linear fractional initial value problem of the form [19]

$$
D^{\alpha} y(t)-\lambda y(t)=h(t), \quad t>0,
$$

where $\lambda$ is a constant, subject to the initial conditions

$$
\left[D^{\alpha-k-1} y(t)\right]_{t=0}=b_{k}
$$

for $k=0,1, \ldots, n-1$. Also, we give solutions to linear partial differential equations such as time fractional backward Kolmogorov equation and time fractional-order linear telegraph equation.

The rest of this paper is organized as follows. In Sect. 2, we give some preliminaries and definitions of fractional calculus, some background on the theory of the natural transform, including definitions and important properties of the natural transform, the fractional natural transform method (FNTM), and we present theorems with detailed proofs. In Sect. 3, we present an analysis method of the FNTM and we implement the FNTM to some applications of LFODE and LFPDE. In Sect. 4, we present tables for different values of $\alpha, x$, and $t$. Section 5 is for discussion and conclusions of this paper.

\section{Background of fractional calculus}

In this section, we give some of the main definitions and notations related to fractional calculus $[7,8,12,17,20,22]$.

Definition 2.1 A real function $f(x), x>0$, is said to be in the space $C_{\mu}, \mu \in \mathbb{R}$, if there exists a real number $q(>\mu)$ such that $f(x)=x^{q} g(x)$, where $g(x) \in C[0, \infty)$, and it is said to be in the space $C_{\mu}^{m}$ if $f^{(m)} \in C_{\mu}, m \in \mathbb{N}$.

Definition 2.2 For an integrable function $f \in C_{\mu}$, the Riemann-Liouville fractional integral operator of order $\alpha \geq 0$ is defined as

$$
\left\{\begin{array}{l}
J^{\alpha} f(x)=\frac{1}{\Gamma(\alpha)} \int_{0}^{x}(x-t)^{\alpha-1} f(t) d t \quad \text { when } \alpha>0, x>0, \\
J^{0} f(x)=f(x) .
\end{array}\right.
$$


Caputo and Mainardi [8] presented a modified fractional differential operator $D^{\alpha}$ in their work on the theory of viscoelasticity to overcome the disadvantages of the RiemannLiouville derivative when someone tries to model real-world problems.

Definition 2.3 The fractional derivative of $f \in C_{-1}^{m}, m \in \mathbb{N}$, in the Caputo sense is defined as

$$
\begin{aligned}
D^{\alpha} f(x) & =J^{m-\alpha} D^{m} f(x) \\
& =\frac{1}{\Gamma(m-\alpha)} \int_{0}^{x}(x-t)^{m-\alpha-1} f^{(m)}(t) d t, \quad m-1<\alpha \leq m, x>0 .
\end{aligned}
$$

Definition 2.4 ([21]) A one-parameter function of the Mittag-Leffler type is defined by the series expansion:

$$
E_{\alpha}(z)=\sum_{k=0}^{\infty} \frac{z^{k}}{\Gamma(\alpha k+1)}, \quad \alpha>0, z \in \mathbb{C} .
$$

Definition 2.5 ([21]) A two-parameter function of the Mittag-Leffler type is defined by the series expansion

$$
E_{\alpha, \beta}(z)=\sum_{k=0}^{\infty} \frac{z^{k}}{\Gamma(\alpha k+\beta)}, \quad \alpha>0, \beta>0, z \in \mathbb{C} .
$$

Lemma 2.1 ([17]) If $m-1<\alpha \leq m, m \in \mathbb{N}$, and $f \in C_{\mu}^{m}, \mu \geq-1$, then

$$
\left\{\begin{array}{l}
D^{\alpha} J^{\alpha} f(x)=f(x) \quad \text { if } x>0, \\
J^{\alpha} D^{\alpha} f(x)=f(x)-\sum_{k=0}^{m-1} f^{(k)}\left(0^{+}\right) \frac{x^{k}}{k !} \quad \text { if } m-1<\alpha<m .
\end{array}\right.
$$

We would like to mention here that the Caputo fractional derivative is used because it allows traditional initial and boundary conditions to be included in the formulation of our problem.

Now we present some background about the nature of the natural transform method (NTM). Given a function $f(t), t \in \mathbb{R}$, the general integral transform is defined by $[5,16]$

$$
\Im[f(t)](s)=\int_{-\infty}^{\infty} K(s, t) f(t) d t,
$$

where $K(s, t)$ represents the kernel of the transform, and $s$ is the real (complex) number independent of $t$. Note that when $K(s, t)$ is $e^{-s t}, t J_{n}(s t)$, and $t^{s-1}(s t)$, Eq. (2.3) gives, respectively, the Laplace transform, Hankel transform, and Mellin transform. Now, for $f(t)$, $t \in(-\infty, \infty)$, consider the integral transforms defined by

$$
\Im[f(t)](u)=\int_{-\infty}^{\infty} K(t) f(u t) d t
$$

and

$$
\Im[f(t)](s, u)=\int_{-\infty}^{\infty} K(s, t) f(u t) d t .
$$


It is worth mentioning that when $K(t)=e^{-t}$, Eq. (2.4) gives the integral Sumudu transform, where the parameter $s$ is replaced by $u$. Moreover, for any value of $n$, the generalized Laplace and Sumudu transforms are respectively defined by $[5,16]$

$$
\ell[f(t)]=F(s)=s^{n} \int_{0}^{\infty} e^{-s^{n+1} t} f\left(s^{n} t\right) d t
$$

and

$$
\mathbb{S}[f(t)]=G(u)=u^{n} \int_{0}^{\infty} e^{-u^{n} t} f\left(t u^{n+1}\right) d t .
$$

Note that when $n=0$, Eq. (2.6) and Eq. (2.7) are the Laplace and Sumudu transforms, respectively. The natural transform of the function $f(t)$ for $t \in \mathbb{R}$ is defined by $[5,16]$

$$
\mathbb{N}[f(t)]=R(s, u)=\int_{-\infty}^{\infty} e^{-s t} f(u t) d t, \quad s, u \in(-\infty, \infty),
$$

where the variables $s$ and $u$ are the natural transform variables. Note that Eq. (2.8) can be written in the form [16]

$$
\mathbb{N}[f(t)]=R^{-}(s, u)+R^{+}(s, u)
$$

It is worth mentioning that if the function $f(t) H(t)$ is defined on the positive real axis $(0, \infty)$, where $f$ is in the functional space, and

$$
A=\left\{f(t): \exists M, \tau_{1}, \tau_{2}>0 \text {, with }|f(t)|<M e^{\frac{|t|}{\tau_{j}}} \text { for } t \in(-1)^{j} \times[0, \infty), j \in \mathbb{Z}^{+}\right\} .
$$

Then, we define the natural transform (N-transform) as

$$
\mathbb{N}[f(t) H(t)]=\mathbb{N}^{+}[f(t)]=R^{+}(s, u)=\int_{0}^{\infty} e^{-s t} f(u t) d t, \quad s, u \in(0, \infty) .
$$

Note that if $u=1$, then Eq. (2.9) can be reduced to the Laplace transform, and if $s=1$, then Eq. (2.9) can be reduced to the Sumudu transform.

Some basic properties of the $\mathrm{N}$-transforms are given as follows $[5,16]$.

Property 1 If $\alpha>-1$, then the natural transform of $t^{\alpha}$ is given by $\mathbb{N}^{+}\left[t^{\alpha}\right]=\frac{\Gamma(\alpha+1) u^{\alpha}}{s^{\alpha+1}}$.

Property 2 If $f^{(n)}(t)$ is the $n$th derivative of the function $f(t)$, then its natural transform is given by $\mathbb{N}^{+}\left[f^{(n)}(t)\right]=\frac{s^{n}}{u^{n}} R(s, u)-\sum_{k=0}^{n-1} \frac{s^{n-(k+1)}}{u^{n-k}} f^{(k)}(0)$.

Property 3 (Natural Sumudu duality (NSD)) If $R(s, u)$ and $G(u)$ are the natural and Sumudu transforms of $f(t) \in A$, respectively, then

$$
\mathbb{N}^{+}[f(t)]=R(s, u)=\frac{1}{s} \int_{0}^{\infty} e^{-t} f\left(\frac{u t}{s}\right) d t=\frac{1}{s} G\left(\frac{u}{s}\right) .
$$

Property 4 (Convolution property) Suppose $F(s, u)$ and $G(s, u)$ are the natural transforms of $f(t)$ and $g(t)$, respectively, both defined in the set $A$. Then the natural transform of their convolution is given by $\mathbb{N}^{+}[(f * g)]=u F(s, u) G(s, u)$. 
Remark 2.1 The convolution of two functions is defined by $f * g=\int_{0}^{t} f(\tau) g(t-\tau) d \tau$.

We now give the proof of Theorems 2.2-2.4.

Theorem 2.2 If $\alpha, \beta>0, a \in \mathbb{R}$, and $\frac{s^{\alpha}}{u^{\alpha}}>|a|$, then we have the inverse natural transform formula

$$
\mathbb{N}^{-1}\left[\frac{u^{\beta-1} s^{\alpha-\beta}}{s^{\alpha}+a u^{\alpha}}\right]=t^{\beta-1} E_{\alpha, \beta}\left(-a t^{\alpha}\right)
$$

Proof First, we take the N-transform of the right-hand side of Eq. (2.10) to get

$$
\begin{aligned}
\mathbb{N}^{+}\left[t^{\beta-1} E_{\alpha, \beta}\left(-a t^{\alpha}\right)\right] & =\frac{1}{u} \int_{0}^{\infty} e^{\frac{-s t}{u}} t^{\beta-1} E_{\alpha, \beta}\left(-a t^{\alpha}\right) d t \\
& =\frac{1}{u} \int_{0}^{\infty} e^{\frac{-s t}{u}} t^{\beta-1} \sum_{k=0}^{\infty} \frac{\left(-a t^{\alpha}\right)^{k}}{\Gamma(\alpha k+\beta)} d t \\
& =\sum_{k=0}^{\infty} \frac{1}{u} \frac{(-a)^{k}}{\Gamma(\alpha k+\beta)} \int_{0}^{\infty} e^{\frac{-s t}{u}} t^{\alpha k+\beta-1} d t .
\end{aligned}
$$

Now we use integration by parts on $\int_{0}^{\infty} e^{\frac{-s t}{u}} t^{\alpha k+\beta-1} d t$ to get

$$
\int_{0}^{\infty} e^{\frac{-s t}{u}} t^{\alpha k+\beta-1} d t=\left(\frac{u}{s}\right)^{\alpha k+\beta} \Gamma(\alpha k+\beta)
$$

Now substitute Eq. (2.12) into Eq. (2.11) to get

$$
\begin{aligned}
\mathbb{N}^{+}\left[t^{\beta-1} E_{\alpha, \beta}\left(-a t^{\alpha}\right)\right] & =\sum_{k=0}^{\infty} \frac{1}{u} \frac{(-a)^{k}}{\Gamma(\alpha k+\beta)}\left(\frac{u}{s}\right)^{\alpha k+\beta} \Gamma(\alpha k+\beta) \\
& =\sum_{k=0}^{\infty} \frac{(-a)^{k}}{s^{\alpha k+\beta}} u^{\alpha k+\beta-1} \\
& =\frac{u^{\beta-1}}{s^{\beta}} \sum_{k=0}^{\infty}\left(\frac{-a u^{\alpha}}{u^{\alpha}}\right)^{k} \\
& =\frac{u^{\beta-1}}{s^{\beta}} \frac{1}{1-\left(\frac{-a u^{\alpha}}{u^{\alpha}}\right)} \\
& =\frac{u^{\beta-1}}{s^{\beta}} \frac{s^{\alpha}}{s^{\alpha}+a u^{\alpha}} .
\end{aligned}
$$

Now the inverse natural transform of Eq. (2.13) is given by

$$
\mathbb{N}^{-1}\left[\frac{u^{\beta-1} s^{\alpha-\beta}}{s^{\alpha}+a u^{\alpha}}\right]=t^{\beta-1} E_{\alpha, \beta}\left(-a t^{\alpha}\right) .
$$

Theorem 2.3 If $\alpha \geq \beta>0, a \in \mathbb{R}$, and $\left(\frac{u}{s}\right)^{\alpha-\beta}>|a|$, then

$$
\mathbb{N}^{-1}\left[\frac{u^{(n+1)(\alpha+\beta)-1}}{\left(s^{\alpha} u^{\beta}+a u^{\alpha} s^{\beta}\right)^{n+1}}\right]=t^{\alpha(n+1)-1} \sum_{k=0}^{\infty} \frac{(-a)^{k}\left(\begin{array}{c}
n+k \\
k
\end{array}\right)}{\Gamma(k(\alpha-\beta)+(n+1) \alpha)} t^{k(\alpha-\beta)} .
$$


Proof Similarly to the proof of Theorem 2.2, we take the N-transform of the right-hand side of Eq. (2.14) to get

$$
\mathbb{N}^{+}\left[t^{\alpha(n+1)-1} \sum_{k=0}^{\infty} \frac{(-a)^{k}\left(\begin{array}{l}
n+k \\
k
\end{array}\right)}{\Gamma(k(\alpha-\beta)+(n+1) \alpha)} t^{k(\alpha-\beta)}\right]=\frac{u^{(n+1)(\alpha+\beta)-1}}{\left(s^{\alpha} u^{\beta}+a u^{\alpha} s^{\beta}\right)^{n+1}} .
$$

Taking the inverse natural transform of Eq. (2.15), we get

$$
\mathbb{N}^{-1}\left[\frac{u^{(n+1)(\alpha+\beta)-1}}{\left(s^{\alpha} u^{\beta}+a u^{\alpha} s^{\beta}\right)^{n+1}}\right]=t^{\alpha(n+1)-1} \sum_{k=0}^{\infty} \frac{(-a)^{k}\left(\begin{array}{c}
n+k \\
k
\end{array}\right)}{\Gamma(k(\alpha-\beta)+(n+1) \alpha)} t^{k(\alpha-\beta)} .
$$

Theorem 2.4 If $\alpha \geq \beta, \alpha>\gamma, a \in \mathbb{R},\left(\frac{u}{s}\right)^{\alpha-\beta}>|a|$, and $\left|\frac{b u^{\alpha+\beta}}{s^{\alpha} u^{\beta}+a s^{\beta} u^{\alpha}}\right|<1$, then

$$
\begin{aligned}
\mathbb{N}^{-1} & {\left[\frac{u^{\alpha-(\gamma+1)+\beta} s^{\gamma}}{s^{\alpha} u^{\beta}+a u^{\alpha} s^{\beta}+b u^{\alpha+\beta}}\right] } \\
& =t^{\alpha-(\gamma+1)} \sum_{n=0}^{\infty} \sum_{k=0}^{\infty} \frac{(-b)^{n}(-a)^{k}\left(\begin{array}{l}
n+k \\
k
\end{array}\right)}{\Gamma(k(\alpha-\beta)+(n+1) \alpha-\gamma)} t^{k(\alpha-\beta)+n \alpha} .
\end{aligned}
$$

Proof Take the N-transform of the right-hand side of Eq. (2.16) to get

$$
\begin{aligned}
& \mathbb{N}^{+}\left[t^{\alpha-(\gamma+1)} \sum_{n=0}^{\infty} \sum_{k=0}^{\infty} \frac{(-b)^{n}(-a)^{k}\left(\begin{array}{c}
n+k \\
k
\end{array}\right)}{\Gamma(k(\alpha-\beta)+(n+1) \alpha-\gamma)} t^{k(\alpha-\beta)+n \alpha}\right] \\
& \quad=\frac{s^{\gamma} u^{\alpha-(\gamma+1)+\beta}}{s^{\alpha} u^{\beta}+a s^{\beta} u^{\alpha}+b u^{\alpha+\beta}} .
\end{aligned}
$$

Taking the inverse N-transform of Eq. (2.17), we get

$$
\begin{aligned}
\mathbb{N}^{-1} & {\left[\frac{u^{\alpha-(\gamma+1)+\beta} s^{\gamma}}{s^{\alpha} u^{\beta}+a u^{\alpha} s^{\beta}+b u^{\alpha+\beta}}\right] } \\
& =t^{\alpha-(\gamma+1)} \sum_{n=0}^{\infty} \sum_{k=0}^{\infty} \frac{(-b)^{n}(-a)^{k}\left(\begin{array}{c}
n+k \\
k
\end{array}\right)}{\Gamma(k(\alpha-\beta)+(n+1) \alpha-\gamma)} t^{k(\alpha-\beta)+n \alpha} .
\end{aligned}
$$

Remark 2.2 We should mention that the proof of Theorems 2.5-2.7 can be found in [25].

Theorem 2.5 If $R(s, u)$ is the natural transform of $f(t)$, then the natural transform of the Riemann-Liouville fractional integral for the function $f(t)$ of order $\alpha$ denoted by $J^{\alpha}[f(t)]$ is given by

$$
\mathbb{N}^{+}\left[J^{\alpha} f(t)\right]=\frac{u^{\alpha}}{s^{\alpha}} R(s, u) .
$$

Theorem 2.6 If $n$ is any positive integer, $n-1 \leq \alpha<n$, and $R(s, u)$ is the natural transform of a function $f(t)$, then the natural transform $R_{\alpha}(s, u)$ of the Riemann-Liouville fractional derivative of the function $f(t)$ of order $\alpha$ denoted by $D^{\alpha} f(t)$ is given by

$$
\mathbb{N}^{+}\left[D^{\alpha} f(t)\right]=R_{\alpha}(s, u)=\frac{s^{\alpha}}{u^{\alpha}} R(s, u)-\sum_{k=0}^{n-1} \frac{s^{k}}{u^{k+1}}\left(D^{\alpha-k-1} f(t)\right)_{t=0} .
$$


Theorem 2.7 If $n$ is any positive integer, $n-1 \leq \alpha<n$, and $R(s, u)$ is the natural transform of a function $f(t)$, then the natural transform $R_{\alpha}^{c}(s, u)$ of the Caputo fractional derivative of the function $f(t)$ of order $\alpha$ denoted by ${ }^{c} D^{\alpha} f(t)$ is given by

$$
\mathbb{N}^{+}\left[{ }^{c} D^{\alpha} f(t)\right]=R_{\alpha}^{c}(s, u)=\frac{s^{\alpha}}{u^{\alpha}} R(s, u)-\sum_{k=0}^{n-1} \frac{s^{\alpha-(k+1)}}{u^{\alpha-k}}\left[D^{k} f(t)\right]_{t=0} .
$$

\section{Analysis of the IFNTM}

In this section, we illustrate the fractional natural transform method (IFNTM) algorithm. Methodology of IFNTM for LFODE:

Consider the linear fractional ordinary differential equation (LFODE) of the form

$$
D^{\alpha} y(t)=y^{\prime \prime}(t)+y^{\prime}(t)+y(t)+k, \quad n-1<\alpha \leq n,
$$

where $k$ is a constant, subject to the initial condition

$$
y(0)=y_{0},
$$

where $D^{\alpha} y(t)$ is the Caputo fractional derivative of the function $y(t)$.

Now we apply the N-transform and Theorem 2.7 to Eq. (3.1) to get

$$
\mathbb{N}^{+}\left[D^{\alpha} y(t)\right]=\mathbb{N}^{+}\left[y^{\prime \prime}(t)+y^{\prime}(t)+y(t)+k\right]
$$

Apply Theorem 2.7 and Property 2 to Eq. (3.3) and Eq. (3.2) to get

$$
\begin{aligned}
& \frac{s^{\alpha}}{u^{\alpha}}\left[\mathbb{N}^{+}[y(t)]-\sum_{k=0}^{n-1} \frac{s^{k-\alpha}}{u^{k+1-\alpha}}\left[D^{\alpha-k-1} y(t)\right]_{t=0}\right] \\
& \quad=\frac{s^{2}}{u^{2}} \mathbb{N}^{+}[y(t)]-\frac{s y(0)}{u^{2}}-\frac{y^{\prime}(0)}{u}+\frac{s}{u} \mathbb{N}^{+}[y(t)]-\frac{y(0)}{u}+\mathbb{N}^{+}[y(t)]+\frac{k}{s} .
\end{aligned}
$$

Then Eq. (3.4) becomes

$$
\begin{aligned}
\mathbb{N}^{+} & {[y(t)]-\sum_{k=0}^{n-1} \frac{s^{k-\alpha}}{u^{k+1-\alpha}}\left[D^{\alpha-k-1} y(t)\right]_{t=0} } \\
= & \frac{u^{\alpha-2}}{s^{\alpha-2}} \mathbb{N}^{+}[y(t)]-\frac{u^{\alpha-2} y_{0}}{s^{\alpha-1}}-\frac{u^{\alpha-1} y^{\prime}(0)}{s^{\alpha}}+\frac{u^{\alpha-1}}{s^{\alpha-1}} \mathbb{N}^{+}[y(t)] \\
& -\frac{u^{\alpha-1} y_{0}}{s^{\alpha}}+\frac{u^{\alpha}}{s^{\alpha}} \mathbb{N}^{+}[y(t)]+\frac{u^{\alpha} k}{s^{\alpha+1}} .
\end{aligned}
$$

Thus

$$
\begin{aligned}
\mathbb{N}^{+} & {[y(t)]-\frac{u^{\alpha-2}}{s^{\alpha-2}} \mathbb{N}^{+}[y(t)]-\frac{u^{\alpha-1}}{s^{\alpha-1}} \mathbb{N}^{+}[y(t)]-\frac{u^{\alpha}}{s^{\alpha}} \mathbb{N}^{+}[y(t)] } \\
& =\sum_{k=0}^{n-1} \frac{s^{k-\alpha}}{u^{k+1-\alpha}}\left[D^{\alpha-k-1} y(t)\right]_{t=0}-\frac{u^{\alpha-2} y_{0}}{s^{\alpha-1}}-\frac{u^{\alpha-1} y^{\prime}(0)}{s^{\alpha}}-\frac{u^{\alpha-1} y_{0}}{s^{\alpha}}+\frac{k u^{\alpha}}{s^{\alpha+1}} .
\end{aligned}
$$


This implies

$$
\mathbb{N}^{+}[y(t)]=\frac{s^{\alpha}}{s^{\alpha}-s^{2} u^{\alpha-2}-s u^{\alpha-1}-u^{\alpha}}\left[g(u, s)-\frac{u^{\alpha-2} y_{0}}{s^{\alpha-1}}-\frac{u^{\alpha-1} y_{0}}{s^{\alpha}}+\frac{k u^{\alpha}}{s^{\alpha+1}}\right],
$$

where $g(u, s)$ is described by

$$
\sum_{k=0}^{n-1} \frac{s^{k-\alpha}}{u^{k+1-\alpha}}\left[D^{\alpha-k-1} y(t)\right]_{t=0}-\frac{u^{\alpha-1} y^{\prime}(0)}{s^{\alpha}}
$$

Taking the natural transform inverse of Eq. (3.7), the solution of Eq. (3.1) is given by

$$
y(t)=\mathbb{N}^{-1}\left[\frac{s^{\alpha}}{s^{\alpha}-s^{2} u^{\alpha-2}-s u^{\alpha-1}-u^{\alpha}}\left[g(u, s)-\frac{u^{\alpha-2} y_{0}}{s^{\alpha-1}}-\frac{u^{\alpha-1} y_{0}}{s^{\alpha}}+\frac{k u^{\alpha}}{s^{\alpha+1}}\right]\right] .
$$

Methodology of IFNTM for LFPDE:

Consider the linear fractional partial differential equation (LFPDE) of the form

$$
D_{t}^{\alpha} v(x, t)+R v(x, t)=g(x, t)
$$

with $0<\alpha \leq 1$, subject to the initial condition

$$
v(x, 0)=h(x)
$$

where $R$ is a linear differential operator, and $g(x, t)$ is a nonhomogeneous term.

We apply the $\mathrm{N}$-transform and Theorem 2.7 to Eq. (3.8) to get

$$
\mathbb{N}^{+}[v(x, t)]=\frac{u^{\alpha}}{s^{\alpha}} \sum_{k=0}^{n-1} \frac{u^{k-\alpha}}{s^{k+1-\alpha}}\left[D^{k} v(x, t)\right]_{t=0}+\frac{u^{\alpha}}{s^{\alpha}} \mathbb{N}^{+}[g(x, t)]-\frac{u^{\alpha}}{s^{\alpha}} \mathbb{N}^{+}[R v(x, t)]
$$

By substituting Eq. (3.9) into Eq. (3.10) and taking the inverse of N-transform of Eq. (3.10) we get

$$
\begin{aligned}
v(x, t) & =\mathbb{N}^{-1}\left[\frac{1}{s} h(x, t)\right]+\mathbb{N}^{-1}\left[\frac{u^{\alpha}}{s^{\alpha}} \mathbb{N}^{+}[g(x, t)]\right]-\mathbb{N}^{-1}\left[\frac{u^{\alpha}}{s^{\alpha}} \mathbb{N}^{+}[R v(x, t)]\right] \\
& =G(x, t)-\mathbb{N}^{-1}\left[\frac{u^{\alpha}}{s^{\alpha}} \mathbb{N}^{+}[R v(x, t)]\right] .
\end{aligned}
$$

Note that $G(x, t)$ arises from the nonhomogeneous term and the prescribed initial conditions. We assume an infinite series solution of the unknown function $v(x, t)$ of the form

$$
v(x, t)=\sum_{n=0}^{\infty} v_{n}(x, t)
$$

Now, we substitute Eq. (3.12) into Eq. (3.11) to get

$$
\sum_{n=0}^{\infty} v_{n}(x, t)=G(x, t)-\mathbb{N}^{-1}\left[\frac{u^{\alpha}}{s^{\alpha}} \mathbb{N}^{+}\left[R \sum_{n=0}^{\infty} v_{n}(x, t)\right]\right]
$$


Comparing both sides of Eq. (3.13), we have:

$$
\begin{aligned}
& v_{0}(x, t)=G(x, t), \\
& v_{1}(x, t)=-\mathbb{N}^{-1}\left[\frac{u^{\alpha}}{s^{\alpha}} \mathbb{N}^{+}\left[R v_{0}(x, t)\right]\right], \\
& v_{2}(x, t)=-\mathbb{N}^{-1}\left[\frac{u^{\alpha}}{s^{\alpha}} \mathbb{N}^{+}\left[R v_{1}(x, t)\right]\right], \\
& v_{3}(x, t)=-\mathbb{N}^{-1}\left[\frac{u^{\alpha}}{s^{\alpha}} \mathbb{N}^{+}\left[R v_{2}(x, t)\right]\right] .
\end{aligned}
$$

Eventually, we have the general recursive relation

$$
v_{n+1}(x, t)=-\mathbb{N}^{-1}\left[\frac{u^{\alpha}}{s^{\alpha}} \mathbb{N}^{+}\left[R v_{n}(x, t)\right]\right], \quad n \geq 1 .
$$

Finally, the approximate solution is given by

$$
v(x, t)=\sum_{n=0}^{\infty} v_{n}(x, t)
$$

Now, we implement the IFNTM to two applications of LFODE and two examples of LFPDE. Then we compare our solutions to the known exact solutions.

Example 3.1 Consider the linear fractional initial value problem of the form [19]

$$
D^{1 / 2} f(t)+f(t)=0,
$$

subject to the initial condition

$$
\left[D^{-1 / 2} f(t)\right]_{t=0}=2 .
$$

Solution: Applying the N-transform to both sides of Eq. (3.15), we obtain

$$
\mathbb{N}^{+}\left[D^{1 / 2} f(t)\right]+\mathbb{N}^{+}[f(t)]=0 .
$$

Thus

$$
\frac{s^{1 / 2}}{u^{1 / 2}} \mathbb{N}^{+}[f(t)]-\sum_{k=0}^{n-1} \frac{s^{k}}{u^{k+1}}\left[D^{-k-1 / 2} f(t)\right]_{t=0}+\mathbb{N}^{+}[f(t)]=0 .
$$

Substitute Eq. (3.16) into Eq. (3.18) to get

$$
\frac{s^{1 / 2}}{u^{1 / 2}} \mathbb{N}^{+}[f(t)]-\frac{2}{u}+\mathbb{N}^{+}[f(t)]=0 .
$$

Now we take the inverse $\mathrm{N}$-transform of Eq. (3.19) and use Theorem 2.7 when $\alpha=\frac{1}{2}, \beta=\frac{1}{2}$, and $a=1$ to get

$$
f(t)=2 t^{-1 / 2} E_{\frac{1}{2}, \frac{1}{2}}\left(-t^{1 / 2}\right) .
$$


Example 3.2 Consider the linear fractional initial value problem of the form [19]

$$
D^{\alpha} y(t)-\lambda y(t)=h(t), \quad t>0
$$

where $\lambda$ is a constant, subject to the initial conditions

$$
\left[D^{\alpha-k-1} y(t)\right]_{t=0}=b_{k} \quad \text { for } k=0,1, \ldots, n-1 .
$$

Solution: Applying the N-transform to both sides of Eq. (3.20), we obtain

$$
\mathbb{N}^{+}\left[D^{\alpha} y(t)\right]-\lambda \mathbb{N}^{+}[y(t)]=\mathbb{N}^{+}[h(t)]
$$

Applying Theorem 2.7 to Eq. (3.22), we get

$$
\frac{s^{\alpha}}{u^{\alpha}} \mathbb{N}^{+}[y(t)]-\sum_{k=0}^{n-1} \frac{s^{k}}{u^{k+1}}\left[D^{\alpha-k-1} y(t)\right]_{t=0}-\lambda \mathbb{N}^{+}[f(t)]=\mathbb{N}^{+}[h(t)]
$$

Substituting Eq. (3.21) into Eq. (3.23), we get

$$
\left[\frac{s^{\alpha}}{u^{\alpha}}-\lambda\right] \mathbb{N}^{+}[y(t)]-\sum_{k=0}^{n-1} \frac{s^{k}}{u^{k+1}} b_{k}=\mathbb{N}^{+}[h(t)]
$$

Then Eq. (3.24) becomes

$$
\mathbb{N}^{+}[y(t)]=\frac{u^{\alpha} \mathbb{N}^{+}[h(t)]}{s^{\alpha}-\lambda u^{\alpha}}+\sum_{k=0}^{n-1} \frac{s^{k} u^{\alpha}}{u^{k+1}\left(s^{\alpha}-\lambda u^{\alpha}\right)} b_{k} .
$$

Simplify the first and second terms of Eq. (3.25):

$$
\frac{u^{\alpha} \mathbb{N}^{+}[h(t)]}{s^{\alpha}-\lambda u^{\alpha}}=u \mathbb{N}^{+}[h(t)] \frac{u^{\alpha-1}}{s^{\alpha}-\lambda u^{\alpha}}
$$

Thus

$$
\begin{aligned}
\sum_{k=0}^{n-1} \frac{s^{k} u^{\alpha}}{u^{k+1}\left(s^{\alpha}-\lambda u^{\alpha}\right)} b_{k} & =\sum_{k=0}^{n-1} \mathbb{N}^{+}\left[b_{k} t^{\alpha-k-1} E_{\alpha, \alpha-k}\left(\lambda t^{\alpha}\right)\right] \\
& =\sum_{k=0}^{n-1} b_{k} \mathbb{N}^{+}\left[t^{\alpha-k-1} E_{\alpha, \alpha-k}\left(\lambda t^{\alpha}\right)\right] .
\end{aligned}
$$

Substituting Eq. (3.26) and Eq. (3.27) into Eq. (3.25), we get

$$
\mathbb{N}^{+}[y(t)]=\mathbb{N}^{+}\left[h(t) * t^{\alpha-1} E_{\alpha, \alpha}\left(\lambda t^{\alpha}\right)\right]+\sum_{k=0}^{n-1} b_{k} \mathbb{N}^{+}\left[t^{\alpha-k-1} E_{\alpha, \alpha-k}\left(\lambda t^{\alpha}\right)\right] .
$$

Now we take the inverse N-transform of Eq. (3.28) to obtain

$$
y(t)=\int_{0}^{\infty}(t-\tau)^{\alpha-1} E_{\alpha, \alpha}\left(\lambda(t-\tau)^{\alpha}\right) h(\tau) d \tau+\sum_{k=0}^{n-1} b_{k} t^{\alpha-k-1} E_{\alpha, \alpha-k}\left(\lambda t^{\alpha}\right) .
$$

This is the exact solution of Eq. (3.20). 


\section{Applications of LFPDE:}

Now we consider two applications of LFPDE, namely the backward Kolmogorov equations in fractal space-time and the time-fractional-order linear telegraph equation.

Example 3.3 Consider the time-fractional backward Kolmogorov equation of the form [13]

$$
D_{t}^{\alpha} v(x, t)=-x^{2} e^{t} v_{x x}(x, t)+(x+1) v_{x}(x, t)+t x, \quad t>0,0<\alpha \leq 1,
$$

subject to the initial condition

$$
v(x, 0)=x+1 .
$$

\section{Solution:}

Applying the N-transform to Eq. (3.29), we obtain

$$
\mathbb{N}^{+}\left[D_{t}^{\alpha} v(x, t)\right]=\mathbb{N}^{+}\left[-x^{2} e^{t} v_{x x}(x, t)+(x+1) v_{x}(x, t)+t x\right]
$$

Using Theorem 2.7 and Eq. (3.30), we get

$$
\begin{aligned}
\mathbb{N}^{+}[v(x, t)] & =\sum_{k=0}^{n-1} \frac{u^{k}}{s^{k+1}}\left[D_{t}^{k} v(x, t)\right]_{t=0}+\frac{u^{\alpha}}{s^{\alpha}} \mathbb{N}^{+}[t x]+\frac{u^{\alpha}}{s^{\alpha}} \mathbb{N}^{+}\left[-x^{2} e^{t} v_{x x}(x, t)+(x+1) v_{x}(x, t)\right] \\
& =\frac{1}{s} v(x, 0)+\frac{x u^{\alpha+1}}{s^{\alpha+2}}+\frac{u^{\alpha}}{s^{\alpha}} \mathbb{N}^{+}\left[-x^{2} e^{t} v_{x x}(x, t)+(x+1) v_{x}(x, t)\right] \\
& =\frac{x+1}{s}+\frac{x u^{\alpha+1}}{s^{\alpha+2}}+\frac{u^{\alpha}}{s^{\alpha}} \mathbb{N}^{+}\left[-x^{2} e^{t} v_{x x}(x, t)+(x+1) v_{x}(x, t)\right] .
\end{aligned}
$$

Now we apply the inverse N-transform to Eq. (3.32) to obtain

$$
v(x, t)=(x+1)+\frac{x t^{\alpha+1}}{\Gamma(\alpha+2)}+\mathbb{N}^{-1}\left[\frac{u^{\alpha}}{s^{\alpha}} \mathbb{N}^{+}\left[-x^{2} e^{t} v_{x x}(x, t)+(x+1) v_{x}(x, t)\right]\right] .
$$

We assume an infinite series solution of the unknown function $v(x, t)$ of the form

$$
v(x, t)=\sum_{n=0}^{\infty} v_{n}(x, t)
$$

Using Eq. (3.33), we rewrite Eq. (3.34) in the form

$$
\begin{aligned}
\sum_{n=0}^{\infty} v_{n}(x, t)= & (x+1)+\frac{x t^{\alpha+1}}{\Gamma(\alpha+2)} \\
& +\mathbb{N}^{-1}\left[\frac{u^{\alpha}}{s^{\alpha}} \mathbb{N}^{+}\left[-x^{2} e^{t} \sum_{n=0}^{\infty} v_{n x x}(x, t)+(x+1) \sum_{n=0}^{\infty} v_{n x}(x, t)\right]\right] .
\end{aligned}
$$

Comparing both sides of Eq. (3.35), we conclude:

$$
v_{0}(x, t)=(x+1)+\frac{x t^{\alpha+1}}{\Gamma(\alpha+2)},
$$




$$
\begin{aligned}
& v_{1}(x, t)=\mathbb{N}^{-1}\left[\frac{u^{\alpha}}{s^{\alpha}} \mathbb{N}^{+}\left[-x^{2} e^{t} v_{0 x x}(x, t)+(x+1) v_{0 x}(x, t)\right]\right], \\
& v_{2}(x, t)=\mathbb{N}^{-1}\left[\frac{u^{\alpha}}{s^{\alpha}} \mathbb{N}^{+}\left[-x^{2} e^{t} v_{1 x x}(x, t)+(x+1) v_{1 x}(x, t)\right]\right], \\
& v_{3}(x, t)=\mathbb{N}^{-1}\left[\frac{u^{\alpha}}{s^{\alpha}} \mathbb{N}^{+}\left[-x^{2} e^{t} v_{2 x x}(x, t)+(x+1) v_{2 x}(x, t)\right]\right] .
\end{aligned}
$$

We continue in this manner to get

$$
v_{n+1}(x, t)=\mathbb{N}^{-1}\left[\frac{u^{\alpha}}{s^{\alpha}} \mathbb{N}^{+}\left[-x^{2} e^{t} v_{n x x}(x, t)+(x+1) v_{n x}(x, t)\right]\right] .
$$

Then, using Eq. (3.36), we can easily compute the remaining components of the unknown function as follows:

$$
\begin{aligned}
v_{1}(x, t) & =\mathbb{N}^{-1}\left[\frac{u^{\alpha}}{s^{\alpha}} \mathbb{N}^{+}\left[-x^{2} e^{t} v_{0 x x}(x, t)+(x+1) v_{0 x}(x, t)\right]\right] \\
& =\mathbb{N}^{-1}\left[\frac{u^{\alpha}}{s^{\alpha}}(x+1) \mathbb{N}^{+}\left[1+\frac{t^{\alpha+1}}{\Gamma(\alpha+2)}\right]\right] \\
& =\mathbb{N}^{-1}\left[\frac{u^{\alpha}}{s^{\alpha}}(x+1)\left(\frac{1}{s}+\frac{u^{\alpha+1}}{s^{\alpha+2}}\right)\right]=(x+1)\left[\frac{t^{\alpha}}{\Gamma(\alpha+1)}+\frac{t^{2 \alpha+1}}{\Gamma(2 \alpha+2)}\right] .
\end{aligned}
$$

Similarly,

$$
\begin{aligned}
v_{2}(x, t) & =\mathbb{N}^{-1}\left[\frac{u^{\alpha}}{s^{\alpha}} \mathbb{N}^{+}\left[-x^{2} e^{t} v_{1 x x}(x, t)+(x+1) v_{1 x}(x, t)\right]\right] \\
& =\mathbb{N}^{-1}\left[\frac{u^{\alpha}}{s^{\alpha}}(x+1) \mathbb{N}^{+}\left[\frac{t^{\alpha}}{\Gamma(\alpha+1)}+\frac{t^{2 \alpha+1}}{\Gamma(2 \alpha+2)}\right]\right] \\
& =\mathbb{N}^{-1}\left[\frac{u^{\alpha}}{s^{\alpha}}(x+1)\left(\frac{u^{\alpha}}{s^{\alpha+1}}+\frac{u^{2 \alpha+1}}{s^{2 \alpha+2}}\right)\right]=(x+1)\left[\frac{t^{2 \alpha}}{\Gamma(2 \alpha+1)}+\frac{t^{3 \alpha+1}}{\Gamma(3 \alpha+2)}\right], \\
v_{3}(x, t) & =\mathbb{N}^{-1}\left[\frac{u^{\alpha}}{s^{\alpha}} \mathbb{N}^{+}\left[-x^{2} e^{t} v_{2 x x}(x, t)+(x+1) v_{2 x}(x, t)\right]\right] \\
& =\mathbb{N}^{-1}\left[\frac{u^{\alpha}}{s^{\alpha}}(x+1)\left(\frac{u^{2 \alpha}}{s^{2 \alpha+1}}+\frac{u^{3 \alpha+1}}{s^{3 \alpha+2}}\right)\right]=(x+1)\left[\frac{t^{3 \alpha}}{\Gamma(3 \alpha+1)}+\frac{t^{4 \alpha+1}}{\Gamma(4 \alpha+2)}\right], \\
v_{4}(x, t) & =(x+1)\left[\frac{t^{4 \alpha}}{\Gamma(4 \alpha+1)}+\frac{t^{5 \alpha+1}}{\Gamma(5 \alpha+2)}\right],
\end{aligned}
$$

and

$$
\nu_{5}(x, t)=(x+1)\left[\frac{t^{5 \alpha}}{\Gamma(5 \alpha+1)}+\frac{t^{6 \alpha+1}}{\Gamma(6 \alpha+2)}\right] .
$$

Hence, the approximate solution of the unknown function $v(x, t)$ is given by

$$
\begin{aligned}
v(x, t) & =\sum_{n=0}^{\infty} v_{n}(x, t) \\
& =v_{0}(x, t)+v_{1}(x, t)+v_{2}(x, t)+v_{3}(x, t)+\cdots
\end{aligned}
$$




$$
\begin{aligned}
= & (x+1)+\frac{x t^{\alpha+1}}{\Gamma(\alpha+2)}+(x+1)\left[\frac{t^{\alpha}}{\Gamma(\alpha+1)}+\frac{t^{2 \alpha+1}}{\Gamma(2 \alpha+2)}\right] \\
& +(x+1)\left[\frac{t^{2 \alpha}}{\Gamma(2 \alpha+1)}+\frac{t^{3 \alpha+1}}{\Gamma(3 \alpha+2)}\right]+(x+1)\left[\frac{t^{3 \alpha}}{\Gamma(3 \alpha+1)}+\frac{t^{4 \alpha+1}}{\Gamma(4 \alpha+2)}\right] \\
& +\cdots
\end{aligned}
$$

Choosing $\alpha=1$ and using Taylor series expansion, Eq. (3.37) becomes:

$$
\begin{aligned}
v(x, t)= & (x+1)+\frac{x t^{2}}{\Gamma(3)}+(x+1)\left[\frac{t}{\Gamma(2)}+\frac{t^{3}}{\Gamma(4)}\right]+(x+1)\left[\frac{t^{2}}{\Gamma(3)}+\frac{t^{4}}{\Gamma(5)}\right] \\
& +(x+1)\left[\frac{t^{3}}{\Gamma(4)}+\frac{t^{5}}{\Gamma(6)}\right]+(x+1)\left[\frac{t^{4}}{\Gamma(5)}+\frac{t^{6}}{\Gamma(7)}\right]+\cdots \\
= & (x+1)+\frac{x t^{2}}{2 !}+(x+1)\left[t+\frac{t^{3}}{3 !}\right]+(x+1)\left[\frac{t^{2}}{2 !}+\frac{t^{4}}{4 !}\right]+(x+1)\left[\frac{t^{3}}{3 !}+\frac{t^{5}}{5 !}\right]+\cdots \\
= & \frac{x t^{2}}{2 !}+(x+1)\left[1+t+\frac{t^{2}}{2 !}+\frac{2 t^{3}}{3 !}+\frac{2 t^{4}}{4 !}+\frac{2 t^{5}}{5 !}+\cdots\right] \\
= & \frac{x t^{2}}{2 !}+(x+1)\left[1+t+\frac{t^{2}}{2 !}+2 \sum_{k=3}^{\infty} \frac{t^{k}}{k !}\right] \\
= & \frac{x t^{2}}{2 !}+(x+1)\left[1+t+\frac{t^{2}}{2 !}+2 \sum_{k=0}^{\infty} \frac{t^{k}}{k !}-2\left(1+t+\frac{t^{2}}{2}\right)\right] \\
= & (x+1)\left[2 e^{t}-1-t\right]-\frac{t^{2}}{2} .
\end{aligned}
$$

This is in fact the exact solution of Eq. (3.29) as in [13].

It is clear from Figs. 1 and 2, the FNTM approximation and the exact solution are in excellent agreement.

Example 3.4 Consider the time-fractional-order linear telegraph equation of the form [27]

$$
D_{t}^{2 \alpha} v(x, t)+2 D_{t}^{\alpha} v(x, t)+v(x, t)=v_{x x}(x, t), \quad 0<\alpha \leq 1,
$$

subject to the initial conditions

$$
v(x, 0)=e^{x} ; \quad v_{t}(x, 0)=-2 e^{x} .
$$
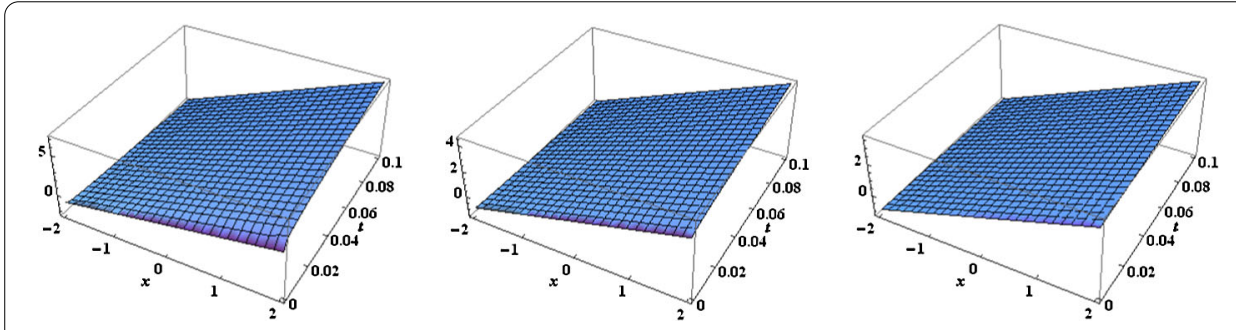

Figure 1 The approximate solutions for Example 3.3 when $\alpha=0.25, \alpha=0.5, \alpha=0.75$, respectively 


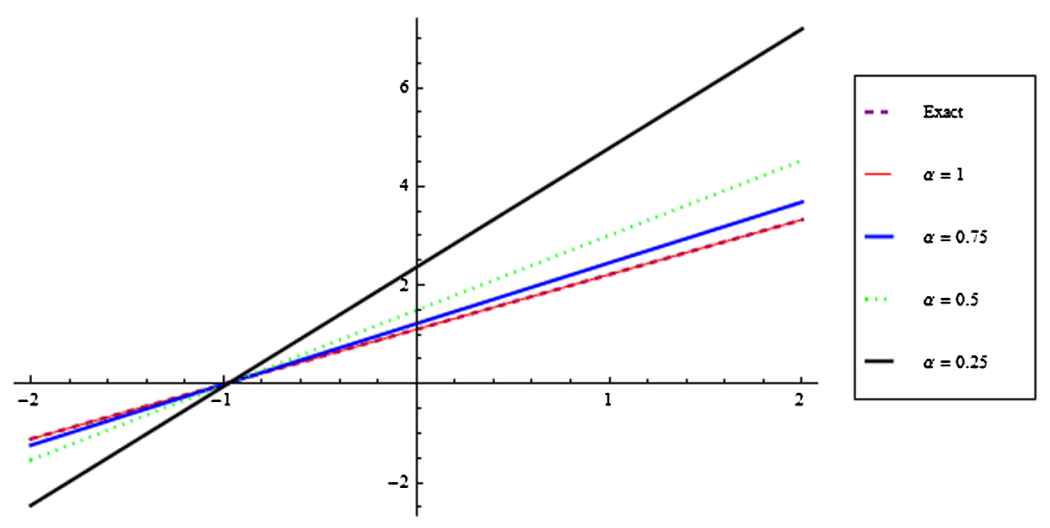

Figure 2 Approximate solutions for Example 3.3 for different values of $\alpha$ when $-2<x<2$

\section{Solution:}

Apply the N-transform to Eq. (3.38) to obtain:

$$
\mathbb{N}^{+}\left[D_{t}^{2 \alpha} v(x, t)\right]+\mathbb{N}^{+}\left[2 D_{t}^{\alpha} v(x, t)+v(x, t)\right]=\mathbb{N}^{+}\left[v_{x x}(x, t)\right] .
$$

Applying Theorem 2.7 to Eq. (3.39), we get

$$
\begin{gathered}
\frac{s^{2 \alpha}}{u^{2 \alpha}} \mathbb{N}^{+}[v(x, t)]-\sum_{k=0}^{n-1} \frac{u^{k-2 \alpha}}{s^{k+1-2 \alpha}}\left[D_{t}^{k} v(x, t)\right]_{t=0}+2 \frac{s^{\alpha}}{u^{\alpha}} \mathbb{N}^{+}[v(x, t)] \\
-2 \sum_{k=0}^{n-1} \frac{u^{k-\alpha}}{s^{k+1-\alpha}}\left[D_{t}^{k} v(x, t)\right]_{t=0}=\mathbb{N}^{+}\left[\frac{\partial^{2}}{\partial t^{2}} v(x, t)-v(x, t)\right] .
\end{gathered}
$$

Note that since $0<2 \alpha \leq 2$, we have two cases.

Case (1): If $0<2 \alpha \leq 1$, then Eq. (3.41) becomes

$$
\mathbb{N}^{+}[v(x, t)]=\frac{1}{s} e^{x}-2 \frac{u^{\alpha}}{s^{\alpha}} \mathbb{N}^{+}[v(x, t)]+2 \frac{u^{\alpha}}{s^{\alpha+1}} e^{x}+\frac{u^{2 \alpha}}{s^{2 \alpha}} \mathbb{N}^{+}\left[v_{x x}(x, t)-v(x, t)\right] .
$$

Now we apply the inverse $\mathrm{N}$-transform to Eq. (3.42) to obtain:

$$
v(x, t)=e^{x}+2 e^{x} \frac{t^{\alpha}}{\Gamma(\alpha+1)}+\mathbb{N}^{-1}\left[\frac{u^{2 \alpha}}{s^{2 \alpha}} \mathbb{N}^{+}\left[v_{x x}(x, t)-v(x, t)\right]-2 \frac{u^{\alpha}}{s^{\alpha}} \mathbb{N}^{+}[v(x, t)]\right] .
$$

We assume an infinite series solution of the unknown function $v(x, t)$ of the form

$$
v(x, t)=\sum_{n=0}^{\infty} v_{n}(x, t)
$$

Using Eq. (3.44), we rewrite Eq. (3.43) in the form

$$
\begin{aligned}
\sum_{n=0}^{\infty} v_{n}(x, t)= & e^{x}\left[1+\frac{2 t^{\alpha}}{\Gamma(\alpha+1)}\right]+\mathbb{N}^{-1}\left[\frac{u^{2 \alpha}}{s^{2 \alpha}} \mathbb{N}^{+}\left[\sum_{n=0}^{\infty} v_{n x x}(x, t)-\sum_{n=0}^{\infty} v_{n}(x, t)\right]\right. \\
& \left.-2 \frac{u^{\alpha}}{s^{\alpha}} \mathbb{N}^{+}\left[\sum_{n=0}^{\infty} v_{n}(x, t)\right]\right] .
\end{aligned}
$$


Comparing both sides of Eq. (3.45), we get

$$
\begin{aligned}
& v_{0}(x, t)=e^{x}\left(1+\frac{2 t^{\alpha}}{\Gamma(\alpha+1)}\right), \\
& v_{1}(x, t)=\mathbb{N}^{-1}\left[\frac{u^{2 \alpha}}{s^{2 \alpha}} \mathbb{N}^{+}\left[v_{0 x x}(x, t)-v_{0}(x, t)\right]-2 \frac{u^{\alpha}}{s^{\alpha}} \mathbb{N}^{+}\left[v_{0}(x, t)\right]\right] \\
& v_{2}(x, t)=\mathbb{N}^{-1}\left[\frac{u^{2 \alpha}}{s^{2 \alpha}} \mathbb{N}^{+}\left[v_{1 x x}(x, t)-v_{1}(x, t)\right]-2 \frac{u^{\alpha}}{s^{\alpha}} \mathbb{N}^{+}\left[v_{1}(x, t)\right]\right] .
\end{aligned}
$$

We continue in this manner to get

$$
v_{n+1}(x, t)=\mathbb{N}^{-1}\left[\frac{u^{2 \alpha}}{s^{2 \alpha}} \mathbb{N}^{+}\left[v_{n x x}(x, t)-v_{n}(x, t)\right]-2 \frac{u^{\alpha}}{s^{\alpha}} \mathbb{N}^{+}\left[v_{n}(x, t)\right]\right] .
$$

Then, using Eq. (3.46), we can easily compute the remaining components of the unknown function as follows:

$$
\begin{aligned}
v_{1}(x, t) & =\mathbb{N}^{-1}\left[\frac{u^{2 \alpha}}{s^{2 \alpha}} \mathbb{N}^{+}\left[v_{0 x x}(x, t)-v_{0}(x, t)\right]-2 \frac{u^{\alpha}}{s^{\alpha}} \mathbb{N}^{+}\left[v_{0}(x, t)\right]\right] \\
& =\mathbb{N}^{-1}\left[-2 \frac{u^{\alpha}}{s^{\alpha}} e^{x} \mathbb{N}^{+}\left[1+\frac{2 t^{\alpha}}{\Gamma(\alpha+1)}\right]\right] \\
& =-2 e^{x}\left(\frac{t^{\alpha}}{\Gamma(\alpha+1)}+\frac{2 t^{2 \alpha}}{\Gamma(2 \alpha+1)}\right) .
\end{aligned}
$$

Similarly,

$$
\begin{aligned}
v_{2}(x, t) & =\mathbb{N}^{-1}\left[\frac{u^{2 \alpha}}{s^{2 \alpha}} \mathbb{N}^{+}\left[v_{1 x x}(x, t)-v_{1}(x, t)\right]-2 \frac{u^{\alpha}}{s^{\alpha}} \mathbb{N}^{+}\left[v_{1}(x, t)\right]\right] \\
& =-2 \mathbb{N}^{-1}\left[-2 \frac{u^{\alpha}}{s^{\alpha}} e^{x} \mathbb{N}^{+}\left[\frac{t^{\alpha}}{\Gamma(\alpha+1)}+\frac{2 t^{2 \alpha}}{\Gamma(2 \alpha+1)}\right]\right] \\
& =4 e^{x}\left(\frac{t^{2 \alpha}}{\Gamma(2 \alpha+1)}+\frac{2 t^{3 \alpha}}{\Gamma(3 \alpha+1)}\right), \\
v_{3}(x, t) & =\mathbb{N}^{-1}\left[\frac{u^{2 \alpha}}{s^{2 \alpha}} \mathbb{N}^{+}\left[v_{2 x x}(x, t)-v_{2}(x, t)\right]-2 \frac{u^{\alpha}}{s^{\alpha}} \mathbb{N}^{+}\left[v_{2}(x, t)\right]\right] \\
& =-2 \mathbb{N}^{-1}\left[\frac{u^{\alpha}}{s^{\alpha}} 4 e^{x} \mathbb{N}^{+}\left[\frac{t^{2 \alpha}}{\Gamma(2 \alpha+1)}+\frac{2 t^{3 \alpha}}{\Gamma(3 \alpha+1)}\right]\right] \\
& =-8 e^{x}\left(\frac{t^{3 \alpha}}{\Gamma(3 \alpha+1)}+\frac{2 t^{4 \alpha}}{\Gamma(4 \alpha+1)}\right), \\
v_{4}(x, t) & =16 e^{x}\left(\frac{t^{4 \alpha}}{\Gamma(4 \alpha+1)}+\frac{2 t^{5 \alpha}}{\Gamma(5 \alpha+1)}\right),
\end{aligned}
$$

and

$$
v_{5}(x, t)=-32 e^{x}\left(\frac{t^{5 \alpha}}{\Gamma(5 \alpha+1)}+\frac{2 t^{6 \alpha}}{\Gamma(6 \alpha+1)}\right) .
$$


Hence, the approximate solution of the unknown function $v(x, t)$ is given by

$$
\begin{aligned}
v(x, t)= & \sum_{n=0}^{\infty} v_{n}(x, t) \\
= & v_{0}(x, t)+v_{1}(x, t)+v_{2}(x, t)+v_{3}(x, t)+\cdots \\
= & e^{x}\left(1+\frac{2 t^{\alpha}}{\Gamma(\alpha+1)}\right)-2 e^{x}\left(\frac{t^{\alpha}}{\Gamma(\alpha+1)}+\frac{2 t^{2 \alpha}}{\Gamma(2 \alpha+1)}\right) \\
& +4 e^{x}\left(\frac{t^{2 \alpha}}{\Gamma(2 \alpha+1)}+\frac{2 t^{3 \alpha}}{\Gamma(3 \alpha+1)}\right) \\
& -8 e^{x}\left(\frac{t^{3 \alpha}}{\Gamma(3 \alpha+1)}+\frac{2 t^{4 \alpha}}{\Gamma(4 \alpha+1)}\right)+16 e^{x}\left(\frac{t^{4 \alpha}}{\Gamma(4 \alpha+1)}+\frac{2 t^{5 \alpha}}{\Gamma(5 \alpha+1)}\right)-\cdots \\
= & e^{x} .
\end{aligned}
$$

Case (2): If $1<2 \alpha \leq 2$, then Eq. (3.41) becomes

$$
\begin{aligned}
\mathbb{N}^{+}[v(x, t)]= & \frac{1}{s} e^{x}+\frac{s^{-2}}{u}\left(-2 e^{x}\right)-2 \frac{u^{\alpha}}{s^{\alpha}} \mathbb{N}^{+}[v(x, t)]+2 \frac{u^{\alpha}}{s^{\alpha+1}} e^{x} \\
& +\frac{u^{2 \alpha}}{s^{2 \alpha}} \mathbb{N}^{+}\left[v_{x x}(x, t)-v(x, t)\right] .
\end{aligned}
$$

Now we apply the inverse N-transform to Eq. (3.48) to obtain:

$$
\begin{aligned}
v(x, t)= & e^{x}-2 e^{x} t+2 e^{x} \frac{t^{\alpha}}{\Gamma(\alpha+1)} \\
& +\mathbb{N}^{-1}\left[\frac{u^{2 \alpha}}{s^{2 \alpha}} \mathbb{N}^{+}\left[v_{x x}(x, t)-v(x, t)\right]-2 \frac{u^{\alpha}}{s^{\alpha}} \mathbb{N}^{+}[v(x, t)]\right] .
\end{aligned}
$$

We assume an infinite series solution of the unknown function $v(x, t)$ of the form

$$
v(x, t)=\sum_{n=0}^{\infty} v_{n}(x, t) .
$$

Using Eq. (3.50), we rewrite Eq. (3.49) in the form

$$
\begin{aligned}
\sum_{n=0}^{\infty} v_{n}(x, t)= & e^{x}\left[1-2 t+\frac{2 t^{\alpha}}{\Gamma(\alpha+1)}\right]+\mathbb{N}^{-1}\left[\frac{u^{2 \alpha}}{s^{2 \alpha}} \mathbb{N}^{+}\left[\sum_{n=0}^{\infty} v_{n x x}(x, t)-\sum_{n=0}^{\infty} v_{n}(x, t)\right]\right. \\
& \left.-2 \frac{u^{\alpha}}{s^{\alpha}} \mathbb{N}^{+}\left[\sum_{n=0}^{\infty} v_{n}(x, t)\right]\right] .
\end{aligned}
$$

Comparing both sides of Eq. (3.51), we conclude:

$$
\begin{aligned}
& v_{0}(x, t)=e^{x}\left(1-2 t+\frac{2 t^{\alpha}}{\Gamma(\alpha+1)}\right), \\
& v_{1}(x, t)=\mathbb{N}^{-1}\left[\frac{u^{2 \alpha}}{s^{2 \alpha}} \mathbb{N}^{+}\left[v_{0 x x}(x, t)-v_{0}(x, t)\right]-2 \frac{u^{\alpha}}{s^{\alpha}} \mathbb{N}^{+}\left[v_{0}(x, t)\right]\right], \\
& v_{2}(x, t)=\mathbb{N}^{-1}\left[\frac{u^{2 \alpha}}{s^{2 \alpha}} \mathbb{N}^{+}\left[v_{1 x x}(x, t)-v_{1}(x, t)\right]-2 \frac{u^{\alpha}}{s^{\alpha}} \mathbb{N}^{+}\left[v_{1}(x, t)\right]\right] .
\end{aligned}
$$


We continue in this manner to get

$$
v_{n+1}(x, t)=\mathbb{N}^{-1}\left[\frac{u^{2 \alpha}}{s^{2 \alpha}} \mathbb{N}^{+}\left[v_{n x x}(x, t)-v_{n}(x, t)\right]-2 \frac{u^{\alpha}}{s^{\alpha}} \mathbb{N}^{+}\left[v_{n}(x, t)\right]\right] .
$$

Then, using Eq. (3.52), we can easily compute the remaining components of the unknown function as follows:

$$
\begin{aligned}
v_{1}(x, t) & =\mathbb{N}^{-1}\left[\frac{u^{2 \alpha}}{s^{2 \alpha}} \mathbb{N}^{+}\left[v_{0 x x}(x, t)-v_{0}(x, t)\right]-2 \frac{u^{\alpha}}{s^{\alpha}} \mathbb{N}^{+}\left[v_{0}(x, t)\right]\right] \\
& =\mathbb{N}^{-1}\left[-2 \frac{u^{\alpha}}{s^{\alpha}} e^{x} \mathbb{N}^{+}\left[1-2 t+\frac{2 t^{\alpha}}{\Gamma(\alpha+1)}\right]\right] \\
& =-2 e^{x}\left(\frac{t^{\alpha}}{\Gamma(\alpha+1)}-\frac{2 t^{\alpha+1}}{\Gamma(\alpha+2)}+\frac{2 t^{2 \alpha}}{\Gamma(2 \alpha+1)}\right) .
\end{aligned}
$$

Similarly,

$$
\begin{aligned}
v_{2}(x, t) & =\mathbb{N}^{-1}\left[\frac{u^{2 \alpha}}{s^{2 \alpha}} \mathbb{N}^{+}\left[v_{1 x x}(x, t)-v_{1}(x t)\right]-2 \frac{u^{\alpha}}{s^{\alpha}} \mathbb{N}^{+}\left[v_{1}(x, t)\right]\right] \\
& =-2 \mathbb{N}^{-1}\left[-2 \frac{u^{\alpha}}{s^{\alpha}} e^{x} \mathbb{N}^{+}\left[\frac{t^{\alpha}}{\Gamma(\alpha+1)}-\frac{2 t^{\alpha+1}}{\Gamma(\alpha+2)}+\frac{2 t^{2 \alpha}}{\Gamma(2 \alpha+1)}\right]\right] \\
& =4 e^{x}\left(\frac{t^{2 \alpha}}{\Gamma(2 \alpha+1)}-\frac{2 t^{2 \alpha+1}}{\Gamma(2 \alpha+2)}+\frac{2 t^{3 \alpha}}{\Gamma(3 \alpha+1)}\right), \\
v_{3}(x, t) & =\mathbb{N}^{-1}\left[\frac{u^{2 \alpha}}{s^{2 \alpha}} \mathbb{N}^{+}\left[v_{2 x x}(x, t)-v_{2}(x, t)\right]-2 \frac{u^{\alpha}}{s^{\alpha}} \mathbb{N}^{+}\left[v_{2}(x, t)\right]\right] \\
& =-2 \mathbb{N}^{-1}\left[\frac{u^{\alpha}}{s^{\alpha}} 4 e^{x} \mathbb{N}^{+}\left[\frac{t^{2 \alpha}}{\Gamma(2 \alpha+1)}-\frac{2 t^{2 \alpha+1}}{\Gamma(2 \alpha+2)}+\frac{2 t^{3 \alpha}}{\Gamma(3 \alpha+1)}\right]\right] \\
& =-8 e^{x}\left(\frac{t^{3 \alpha}}{\Gamma(3 \alpha+1)}-\frac{2 t^{3 \alpha+1}}{\Gamma(3 \alpha+2)}+\frac{2 t^{4 \alpha}}{\Gamma(4 \alpha+1)}\right), \\
v_{4}(x, t) & =16 e^{x}\left(\frac{t^{4 \alpha}}{\Gamma(4 \alpha+1)}-\frac{2 t^{4 \alpha+1}}{\Gamma(4 \alpha+2)}+\frac{2 t^{5 \alpha}}{\Gamma(5 \alpha+1)}\right),
\end{aligned}
$$

and

$$
\nu_{5}(x, t)=-32 e^{x}\left(\frac{t^{5 \alpha}}{\Gamma(5 \alpha+1)}-\frac{2 t^{5 \alpha+1}}{\Gamma(5 \alpha+2)}+\frac{2 t^{6 \alpha}}{\Gamma(6 \alpha+1)}\right) .
$$

Hence, the approximate solution of the unknown function $v(x, t)$ is given by

$$
\begin{aligned}
v(x, t)= & \sum_{n=0}^{\infty} v_{n}(x, t) \\
= & v_{0}(x, t)+v_{1}(x, t)+v_{2}(x, t)+v_{3}(x, t)+\cdots \\
= & e^{x}\left(1-2 t+\frac{2 t^{\alpha}}{\Gamma(\alpha+1)}\right)-2 e^{x}\left(\frac{t^{\alpha}}{\Gamma(\alpha+1)}-\frac{2 t^{\alpha+1}}{\Gamma(\alpha+2)}+\frac{2 t^{2 \alpha}}{\Gamma(2 \alpha+1)}\right) \\
& +4 e^{x}\left(\frac{t^{2 \alpha}}{\Gamma(2 \alpha+1)}-\frac{2 t^{2 \alpha+1}}{\Gamma(2 \alpha+2)}+\frac{2 t^{3 \alpha}}{\Gamma(3 \alpha+1)}\right)
\end{aligned}
$$




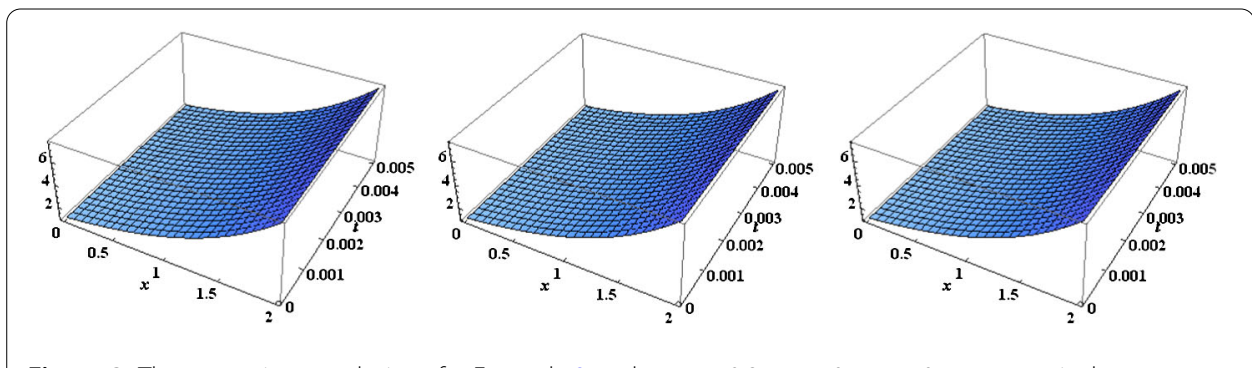

Figure 3 The approximate solutions for Example 3.4 when $\alpha=0.25, \alpha=0.5, \alpha=0.75$, respectively

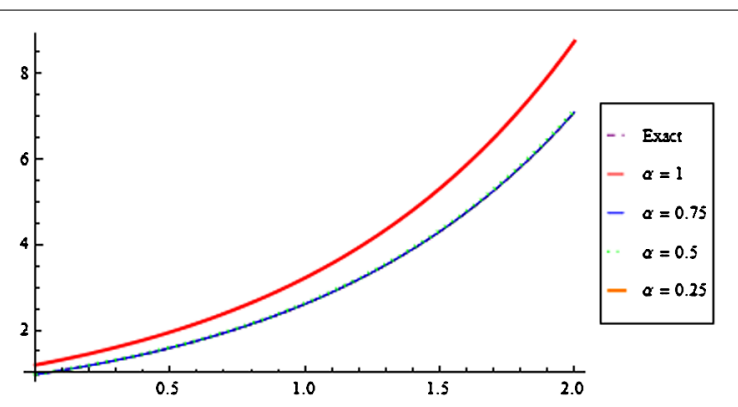

Figure 4 Approximate solutions for Example 3.4 for different values of $\alpha$ when $0<x<2$

$$
\begin{aligned}
& -8 e^{x}\left(\frac{t^{3 \alpha}}{\Gamma(3 \alpha+1)}-\frac{2 t^{3 \alpha+1}}{\Gamma(3 \alpha+2)}+\frac{2 t^{4 \alpha}}{\Gamma(4 \alpha+1)}\right) \\
& +16 e^{x}\left(\frac{t^{4 \alpha}}{\Gamma(4 \alpha+1)}-\frac{2 t^{4 \alpha+1}}{\Gamma(4 \alpha+2)}+\frac{2 t^{5 \alpha}}{\Gamma(5 \alpha+1)}\right)-\cdots \\
= & e^{x}\left(1-2 t+\frac{4 t^{\alpha+1}}{\Gamma(\alpha+2)}-\frac{8 t^{2 \alpha+1}}{\Gamma(2 \alpha+2)}+\frac{16 t^{3 \alpha+1}}{\Gamma(3 \alpha+2)}-\frac{32 t^{4 \alpha+1}}{\Gamma(4 \alpha+2)}+\cdots\right) .
\end{aligned}
$$

Choosing $\alpha=1$ and using Taylor series expansion, Eq. (3.53) becomes

$$
\begin{aligned}
v(x, t) & =e^{x}\left(1-2 t+\frac{4 t^{2}}{\Gamma(3)}-\frac{8 t^{3}}{\Gamma(4)}+\frac{16 t^{4}}{\Gamma(5)}-\frac{32 t^{5}}{\Gamma(6)}+\cdots\right) \\
& =e^{x-2 t} .
\end{aligned}
$$

Hence, this solution is equivalent to the exact solution when $\alpha=1$ in [27].

It is clear from Figs. 3 and 4 that the FNTM approximation and the exact solution are in excellent agreement.

\section{Tables of numerical calculations}

In this section, we calculate the numerical of approximate solutions for Example 3.3 and Example 3.4 for different values of $\alpha$ and different values of $x$ and $t$. In Table 1, we consider different values of $x$ and $t$ and for the same values of $\alpha$. Similarly, in Table 2, we consider different values of $x$ and $t$ for the same values of $\alpha$.

\section{Conclusion}

In this paper, we proved three theorems related to the fractional natural transform method (FNTM), and we successfully applied the new method to obtain solutions to two lin- 
Table 1 The results obtained for Example 3.3 for different values of $\alpha$

\begin{tabular}{|c|c|c|c|c|c|c|}
\hline \multirow[t]{2}{*}{$x$} & \multirow[t]{2}{*}{$t$} & \multirow[t]{2}{*}{$\alpha=0.25$} & \multirow[t]{2}{*}{$\alpha=0.5$} & \multirow[t]{2}{*}{$\alpha=0.75$} & \multicolumn{2}{|l|}{$\alpha=1$} \\
\hline & & & & & Numerical & Exact \\
\hline-2 & $\begin{array}{l}0.02 \\
0.04 \\
0.06 \\
0.08\end{array}$ & $\begin{array}{l}-1.67532 \\
-1.91317 \\
-2.1113 \\
-2.29181\end{array}$ & $\begin{array}{l}-1.1864 \\
-1.28553 \\
-1.37373 \\
-1.45781\end{array}$ & $\begin{array}{l}-1.06139 \\
-1.10818 \\
-1.15302 \\
-1.19766\end{array}$ & $\begin{array}{l}-1.0206 \\
-1.04242 \\
-1.06547 \\
-1.08977\end{array}$ & $\begin{array}{l}-1.0206 \\
-1.04242 \\
-1.06547 \\
-1.08977\end{array}$ \\
\hline 0 & $\begin{array}{l}0.02 \\
0.04 \\
0.06 \\
0.08\end{array}$ & $\begin{array}{l}1.66204 \\
1.8816 \\
2.05888 \\
2.21671\end{array}$ & $\begin{array}{l}1.18214 \\
1.2735 \\
1.35162 \\
1.42376\end{array}$ & $\begin{array}{l}1.06007 \\
1.10373 \\
1.14398 \\
1.1827\end{array}$ & $\begin{array}{l}1.0202 \\
1.04082 \\
1.06187 \\
1.08337\end{array}$ & $\begin{array}{l}1.0202 \\
1.04082 \\
1.06187 \\
1.08337\end{array}$ \\
\hline 2 & $\begin{array}{l}0.02 \\
0.04 \\
0.06 \\
0.08\end{array}$ & $\begin{array}{l}4.9994 \\
5.67637 \\
6.22907 \\
6.72523\end{array}$ & $\begin{array}{l}3.55068 \\
3.83253 \\
4.07698 \\
4.30534\end{array}$ & $\begin{array}{l}3.18154 \\
3.31564 \\
3.44098 \\
3.56306\end{array}$ & $\begin{array}{l}3.06101 \\
3.12406 \\
3.18922 \\
3.25652\end{array}$ & $\begin{array}{l}3.06101 \\
3.12406 \\
3.18922 \\
3.25652\end{array}$ \\
\hline
\end{tabular}

Table 2 The results obtained for Example 3.4 for different values of $\alpha$

\begin{tabular}{lllllll}
\hline$x$ & $t$ & $\alpha=0.25$ & $\alpha=0.5$ & $\alpha=0.75$ & \multicolumn{2}{l}{$\alpha=1$} \\
\cline { 6 - 7 } & & & & & Numerical & Exact \\
\hline 0.5 & 0.001 & 1.64621 & 1.64557 & 1.64545 & 1.64543 & 1.64543 \\
& 0.003 & 1.63568 & 1.6396 & 1.63898 & 1.63886 & 1.63886 \\
& 0.005 & 1.62066 & 1.63386 & 1.63261 & 1.63232 & 1.63232 \\
1 & 0.001 & 2.71414 & 2.71309 & 2.71288 & 2.71285 & 2.71285 \\
& 0.003 & 2.69678 & 2.70324 & 2.70223 & 2.70202 & 2.70202 \\
& 0.005 & 2.67201 & 2.69379 & 2.69172 & 2.69123 & 2.69123 \\
1.5 & 0.001 & 4.47487 & 4.47314 & 4.47279 & 4.47273 & 4.47273 \\
& 0.003 & 4.44623 & 4.45688 & 4.45522 & 4.45488 & 4.45488 \\
& 0.005 & 4.4054 & 4.4413 & 4.4379 & 4.4371 & 4.4371 \\
2 & 0.001 & 7.37781 & 7.37495 & 7.37438 & 7.37429 & 7.37429 \\
& 0.003 & 7.3306 & 7.34816 & 7.34542 & 7.34485 & 7.34485 \\
& 0.005 & 7.26328 & 7.32247 & 7.31686 & 7.31553 & 7.31553 \\
\hline
\end{tabular}

ear fractional ordinary differential equations and two linear fractional partial differential equations. We also found exact solutions to all physical models in the case $\alpha=1$. The FNTM introduces a significant improvement in the fields over existing techniques. Our goal in the future is to apply the FNTM to other fractional linear ODEs and PDEs that arise in other areas of science.

\section{Acknowledgements}

The authors are very grateful to the reviewers and the editor for their constructive comments and valuable suggestions to improve the presentation of the paper.

\section{Funding}

This work was not supported by any source.

Availability of data and materials

Data sharing not applicable to this article as no data sets were generated or analyzed during the current study.

\section{Competing interests}

The authors declare that they have no competing interests.

\section{Authors' contributions}

Both authors contributed equally to the manuscript. Both authors read and approved the final manuscript.

\section{Publisher's Note}

Springer Nature remains neutral with regard to jurisdictional claims in published maps and institutional affiliations. 
Received: 31 January 2018 Accepted: 13 May 2018 Published online: 25 June 2018

\section{References}

1. Adomian, G.: A new approach to nonlinear partial differential equations. J. Math. Anal. Appl. 102, 420-434 (1984)

2. Adomian, G.: Solving Frontier Problems of Physics: The Decomposition Method. Kluwer Academic, Boston (1994)

3. Al-Rabtah, A., Momani, S., Ramadan, M.A.: Solving linear and non-linear fractional differential equations using spline functions. Abstr. Appl. Anal. 2012, Article ID 426514 (2012)

4. Belgacem, F.B.M., Silambarasan, R.: Theoretical investigations of the natural transform. In: Progress in Electromagnetics Research Symposium Proceedings, Suzhou, China, Sept. 12-16 (2011)

5. Belgacem, F.B.M., Silambarasan, R.: Maxwell's equations solutions through the natural transform. Math. Eng. Sci. Aerosp. 3(3), 313-323 (2012)

6. Caputo, M.: Linear models of dissipation whose $Q$ is almost frequency independent. Part II. Geophys. J. Int. 13 529-539 (1967)

7. Caputo, M.: Elasticitae dissipazione. Zanichelli, Bologna (1969)

8. Caputo, M., Linear, M.F.: Models of dissipation in anelastic solids. Riv. Nuovo Cimento 1, 161-168 (1971)

9. Garg, M., Manohar, P.: Numerical solution of fractional diffusion-wave equation with two space variables by matrix method. Fract. Calc. Appl. Anal. 13(2), 191-207 (2010)

10. Garg, M., Sharma, A.: Solution of space-time fractional telegraph equation by Adomian decomposition method. J. Inequal. Spec. Funct. 2(1), 1-7 (2011)

11. He, J.H.: Approximate analytical solution for seepage flow with fractional derivatives in porous media. Comput. Methods Appl. Mech. Eng. 167, 57-68 (1998)

12. Hilfer, R.: Applications of Fractional Calculus in Physics. World Scientific, Singapore (2000)

13. Karbalaie, A., Montazer, M., Muhammed, H.H.: New approach to find the exact solution of fractional partial differential equation. WSEAS Trans. Math. 11(10), 908-917 (2012)

14. Katatbeh, Q.D., Belgacem, F.B.M.: Applications of the Sumudu transform to fractional differential equations. Nonlinear Stud. J. 18(1), 99-112 (2011)

15. Kazem, S.: Exact solution of some linear fractional differential equations by Laplace transform. Int. J. Nonlinear Sci. 16(1), 3-11 (2013)

16. Khan, Z.H., Khan, W.A.: N-transform properties and applications. NUST J. Eng. Sci. 1(1), 127-133 (2008)

17. Kilbas, A.A., Srivastava, H.M., Trujillo, J.J.: Theory and Applications of Fractional Differential Equations. Elsevier, Amsterdam (2006)

18. Kumar, D., Singh, J., An, K.A.: Efficient approach for fractional Harry Dym equation by using Sumudu transform. Abstr. Appl. Anal. 2013, Article ID 608943 (2013)

19. Li, Z.B.: An extended fractional complex transform. Int. J. Nonlinear Sci. Numer. Simul. 11, 335-337 (2010)

20. Miller, K.S., Ross, B.: An Introduction to the Fractional Calculus and Differential Equations. Wiley, New York (1993)

21. Mittag-Leffler, G.M.: Sur la nouvelle fonction $\mathrm{E}_{\alpha}$ (x). C. R. Acad. Sci. Paris 137, 554-558 (1903)

22. Podlubny, I.: Fractional Differential Equations, Mathematics in Science and Engineering. Academic Press, San Diego (1999)

23. Rawashdeh, M.: A new approach to solve the fractional Harry Dym equation using the FRDTM. Int. J. Pure Appl. Math. 95(4), 553-566 (2014)

24. Rawashdeh, M.: An efficient approach for time-fractional damped burger and time-Sharma-Tasso-Olver equations using the FRDTM. Appl. Math. Inf. Sci. 9(3), 1239-1246 (2015)

25. Rawashdeh, M.: The fractional natural decomposition method: theories and applications. Math. Methods Appl. Sci. 40(7), 2362-2376 (2017)

26. Rawashdeh, M., Solving, M.S.: Nonlinear ordinary differential equations using the NDM. J. Appl. Anal. Comput. 5(1), 77-88 (2015)

27. Rawashdeh, M.S.: A reliable method for the space-time fractional Burgers and time-fractional Cahn-Allen equations via the FRDTM. Adv. Differ. Equ. 2017(1), 99 (2017)

28. Rawashdeh, M.S., Al-Jammal, H.: Numerical solutions for systems of nonlinear fractional ordinary differential equations using the FNDM. Mediterr. J. Math. 13(6), 4661-4677 (2016)

29. Rawashdeh, M.S., Al-Jammal, H.: New approximate solutions to fractional nonlinear systems of partial differential equations using the FNDM. Adv. Differ. Equ. 2016(1), 235 (2016)

30. Ray, S.S., Bera, R.K.: An approximate solution of a nonlinear fractional differential equation by Adomian decomposition method. Appl. Math. Comput. 167, 561-571 (2005)

\section{Submit your manuscript to a SpringerOpen ${ }^{\circ}$ journal and benefit from:}

- Convenient online submission

- Rigorous peer review

- Open access: articles freely available online

- High visibility within the field

- Retaining the copyright to your article

Submit your next manuscript at $\boldsymbol{~ s p r i n g e r o p e n . c o m ~}$ 